\section{Mammografie nach brusterhaltender Therapie: Genügt einmal jährlich?}

\section{Zur Nachsorge nach brusterhaltender Therapie (BET) des Mammakarzinoms empfiehlt die Deutsche Krebsgesellschaft in den ersten drei Jahren Mammo- grafien in Halbjahresabständen. Diese Frequenz wird jedoch international diskutiert.}

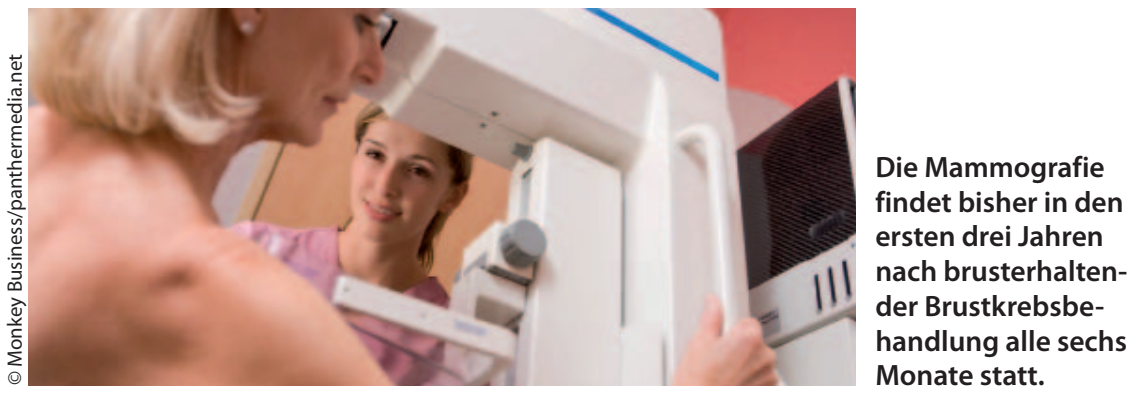

ei der Mammografie nach BET gehen die Empfehlungen der Fachgesellschaften auseinander: Während die American Society of Clinical Oncology (ASCO) die erste postoperative Mammografie ein Jahr nach Diagnose und zumindest sechs Monate nach Beendigung der Bestrahlungstherapie fordert, sieht das National Comprehensive Cancer Network (NCCN) vereinfachend für alle BET-Patientinnen einmal jährliche Röntgenuntersuchungen vor. Nach den Leitlinien der Deutschen Krebsgesellschaft soll dagegen in den ersten drei Jahren alle sechs Monate eine Mammografie durchgeführt werden.

Solche kurzen Screening-Intervalle scheinen jedoch im Hinblick auf Rezidive kaum einen Vorteil zu bringen. Richard V. Hymas und sein Team von der Universität Utah hatten die Detektionsraten bei 1.435 Brustkrebspatientinnen, die nach einem intensiven Protokoll mammografiert worden waren, mit den Ergebnissen des jährlichen Screenings der Allgemeinbevölkerung verglichen. Das im US-Bundesstaat Utah überwiegend eingesetzte „Intermountain-Healthcare“-Protokoll sieht Mammografien nach sechs, zwölf, 18 und 24 Monaten vor, ergänzt durch Röntgenuntersuchungen der kontralateralen Brust zwölf und 24 Monate nach BET.

Bei 23 der „Intermountain“-Patientinnen waren beide Brüste befallen, sodass die BET insgesamt an 1.485 Mammae durchgeführt wurde. Das Staging hatte bei $96 \%$ T2 oder weniger ergeben, $16 \%$ waren duktale In-situ-Karzinome (DCIS). Insgesamt wurden allein durch die Mammografie innerhalb der ersten zwei Jahre nach Diagnose acht ipsilaterale Rezidive identifiziert. Die Forscher ermittelten jeweils die separaten Detektionsraten für das erste und das zweite Jahr nach Diagnose und fanden eine Rate von 1,9 bzw. 4,9 Brustkrebsfällen pro 1.000 mammografierte Brüste. Die „Ausbeute“ des allgemeinen Screenings war im ersten Jahr sogar etwas größer: Die Detektionsrate lag bei 2,3 ( $\mathrm{p}=0,99)$. Im zweiten Jahr war zwar eine leichte Überlegenheit des intensiveren Screenings zu sehen, aber auch hier war die Differenz nicht signifikant $(\mathrm{p}=0,07)$.

Die Forscher betonen, dass die hohe Mammografiefrequenz im ersten Jahr 180 Sonografien, 13 MRTs und 41 Biopsien nach sich gezogen hat. Diese häufigen Prozeduren könnten dazu beitragen, die - oft unbegründete - Rezidivangst der Patientinnen zu steigern, so Hymas. Innerhalb eines so kurzen Zeitfensters könne es zudem schwierig sein, zwischen Narbengewebe als Folge des chirurgischen Eingriffs, bestrahlungsbedingten Gewebsveränderungen und Tumorgewebe $\mathrm{zu}$ unterscheiden.

Fazit: Nach einer brusterhaltenden Krebstherapie seine Mammografien in Jahresabständen eventuell ausreichend, so die Autoren. Dieses Vorgehen rechtfertige sich auch aus dem beobachteten Risikoanstieg vor allem im zweiten Jahr nach der Krebsdiagnose. Neben der Kosteneinsparung läge der Vorteil auch in der geringeren Belastung der Patientinnen, die beide Brüste zeitgleich einmal im Jahr röntgen lassen könnten. Elke Oberhofer

Hymas RV et al. Is short-interval mammography necessary after breast conservation surgery and radiation treatment in breast cancer patients? Int J Radiation Oncol Biol Phys. 2012;83(2):519-24.

Kommentar von Prof. Schreer: Die radiodiagnostische Nachsorgestrategie hat nicht nur zum Ziel, ein intramammäres Rezidiv frühest möglich zu diagnostizieren, sondern auch der Patientin die Sicherheit zu vermitteln, dass alles in Ordnung ist. Dieser Wunsch ist in der frühen Zeit nach der Primärtherapie besonders groß, denn die Patientinnen wissen, dass mit Mammo- und Sonografie meist der Primärtumor erkannt wurde. Für die Diagnostiker ist die erste bildgebende Kontrolle nach abgeschlossener Strahlentherapie wegweisend als Ausgangs-Befunderfassung der postoperativen und poststrahlentherapeutischen Strukturveränderungen. Diese frühen posttherapeutischen Veränderungen müssen bekannt sein und als solche dokumentiert

„Wir sollten nicht von unserer bisherigen Strategie abweichen."

werden für die weitere korrekte Verlaufsbeobachtung und Bildinterpretation. Die Autoren beklagen notwendige Zusatzuntersuchungen, ausgelöst durch Unklarheiten im ersten Jahr nach Therapie. Dies entspricht nicht der Erfahrung in unserem Land, da im Rahmen der bildgebenden Nachsorge in den meisten Fällen eine Sonografie von Mammae und Axillae durchgeführt wird und die MRT nicht vor zwölf Monaten nach Strahlentherapie indiziert wird. Sich nur auf die (geringe) Häufigkeit der entdeckten Rezidive zu konzentrieren, wird der Nachsorge-Aufgabe nicht gerecht. Ich sehe daher keinen Grund, als Folge der vorliegenden Ergebnisse von unserer bisherigen Strategie abzuweichen.

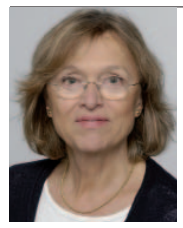

Prof. Dr. med. Ingrid Schreer Mammazentrum, Klinik für Gynäkologie und Geburtshilfe, Universitätsklinikum Schleswig-Holstein, Campus Kiel ischreer@email.uni-kiel.de 